and dislocation/sprain (12\%). In both groups, the cause most often specified was a fall (56\%). ASD cases and POP controls had similar odds of injury (crude odds ratio $[\mathrm{cOR}]=1.1$ [95\% CI: $0.9,1.4]$; adjusted $\mathrm{OR}[\mathrm{aOR}]=1.2[0.9,1.7])$ and serious injury $(\mathrm{cOR}=1.1[0.9,1.4] ; \mathrm{aOR}=1.2[0.9,1.6])$.

Conclusions Children with ASD and population control children had similar odds of any medically-treated injury and serious injury. Sociodemographic and IQ differences did not influence these results. We plan to further explore and compare specific injury types and causes, and additional injury outcomes, between the two groups.

\section{THE DYNAMICS IN LEADING CAUSE OF INJURY MORTALITY BY DEMOGRAPHIC TRAITS IN NINGBO, CHINA: 2004-2013}

${ }^{1}$ Hui Li, ${ }^{1}$ Yinchao Zhu, ${ }^{1}$ Guozhang Xu, ${ }^{1}$ Yong Wang, ${ }^{1}$ Jieping Chen, ${ }^{2}$ Liyuan Han. ${ }^{1}$ Institute of Non-Communicable Disease Control and Prevention, Ningbo Municipal Centre for Disease Control and Prevention, Ningbo, Zhejiang Province, China; ${ }^{2}$ Medical School, Ningbo University ,Ningbo, Zhejiang Province, China

\subsection{6/injuryprev-2016-042156.479}

Background Injuries have emerged as a crucial public health concern in China, accounting for about $10 \%$ of total mortality. Methods Data from the death registry system in Ningbo in the period 2004-2013 were analysed to explore the tendency of injuries, using linear regression model on a log and absolute scale of mortality rate respectively.

Results The average crude injury mortality rate was 56.37/ 100,000 /year, accounting for $9.09 \%$ of all deaths, and showed a substantial downturn (-73.28\% of Annual Percent Change). The ratio of male to female was diminishing and the injury-related deaths were ageing significantly. MV traffic crashes, drowning and suicide had mainly contributed 50.87\%, 18.18\% and $10.52 \%$ for decrease of all-cause injury mortality rate respectively, instead, only fall had contributed $100 \%$ for increase. The greatest reduction occurred in rural-man, with the annual rate changes of $-2.19 / 100,000 /$ year, followed by urban-man $(-1.96 /$ $100,000 /$ year $)$, urban-woman $(-0.75 / 100,000 /$ year $)$ and ruralwoman $(-0.58 / 100,000 /$ year $)$ respectively. Alarmingly, women were disproportionately suffering from fall with a sharp increasing trend, especially in urban-woman. The average crude mortality in adults age 65 and older was 255.98/100,000/year and displayed a increasing trend $(\beta=0.0143, \mathrm{p}=0.0299)$.

Conclusions This study provides a comprehensive overview of the dynamic in injury-related mortality rate in a developed city in China, where some injury patterns are becoming similar with some high-income countries. Appropriate preventive strategies should be urgently initiated to control this aggressive evolution, basing on the successful experiences.

\section{EXERCISE IN TREATMENT AND REHABILITATION OF HIP OSTEOARTHRITIS - A 12-WEEK PILOT STUDY}

${ }^{1}$ Kirsti Uusi-Rasi, ${ }^{1}$ Saija Karinkanta, ${ }^{1}$ Radhika Patil, ${ }^{1,2}$ Pekka Kannus, ${ }^{1}$ Kari Tokola, ${ }^{1}$ Harri Sievänen. ${ }^{1}$ The UKK Institute for Health Promotion Research, Finland; ${ }^{2}$ Tampere University Hospital, Finland

\subsection{6/injuryprev-2016-042156.480}

Background Osteoarthritis (OA) is a chronic joint disease with the hip and knee being commonly affected lower limb sites. There is evidence supporting that aerobic and strength training is beneficial for reducing pain and improving physical function in older adults with mild-to-moderate knee and hip OA. Good physical function is crucial in preventing fall-induced injuries in OA patients.

Methods The aim of this pilot study is to test the safety and feasibility of a tailored exercise program with particular emphasis on maintaining appropriate training intensity while minimising adverse events and injuries.

The self-reported disease-specific pain and physical function will be assessed using the pain and functioning subscales of the WOMAC questionnaire (range $0-100 \mathrm{~mm}$ ) at baseline and at 12 weeks. The maximal leg-extensor strength, dynamic balance, Timed-up and go (TUG), Short physical performance battery (SPPB; includes tests of balance, 4-metre walking speed and 5time chair stand) will be used in assessing physical functioning objectively. In addition, hip range of motion and stair-climb-test (step height $20 \mathrm{~cm}$ ) will be used.

Thirteen women with diagnosed hip OA were recruited and received the exercise program 3 times a week. Main inclusion criteria were age $\geq 65$ years, pain experienced in the hip region and living at home independently.

Results The pilot study started in September 2015, and the end point measurements will be done in December 2015. Dimensions such as intensity progression, individual tailoring of exercises and suitability for varying pain levels and functional ability will be evaluated. In assessing the treatment outcomes, each participant will function as her own control.

Conclusions Training intensity needs to be optimal to insure safety as well as progression in physical function and pain relief. The larger randomised controlled trial will be planned according to experience and feedback received from this pilot study.

\section{THE INJURY MORTALITY MODEL IN SHENZHEN CITY FROM 1994-2013, A RAPIDLY DEVELOPING CITY}

${ }^{1}$ Xiaojian Liu, ${ }^{1}$ Yonsheng Wu, ${ }^{1}$ Yingbin Fu, ${ }^{1}$ Jinquan Cheng, ${ }^{2}$ Liping Li. ${ }^{1}$ Shenzhen Centre for Disease Control and Prevention, China; ${ }^{2}$ Shantou University Medical College, China

\subsection{6/injuryprev-2016-042156.481}

Background In the 20 years, with the rapid development of economy in Shenzhen City, mortality model has been changed. However, we are still not sure the change tendency of injury mortality model, proportion of injury related death, and the main leading injury causes (such as road traffic injury, suicide, and fall). The study is aim to figure out these problems, and teen promote scientific advices for injury prevention.

Methods The injury mortality data of 1994-2006 wwre collected from the Funeral Parlour, and other data of 2007-2013 were collected from the death surveillance system in China. The cause of injury related death was coded from V01 to Y99. All the data were cleaning by uniform standards, and analysed by SPSS 20.0 .

Results Injury mortality rate has been reduced from 3.19/10000 to $1.30 / 10000$ in the 20 years. Injury ratio of all deaths has decreased from $52.1 \%$ in 1994 to $14.8 \%$ in 2013 . The majority of the injury mortality was man, about $70 \%$. The floating population accounted for about $90 \%$ of injury related deaths. Although the ratio of injury death has been descended from $43.5 \%$ to $27.9 \%$ in 20 years, road traffic injury was the first leading cause of injury related death. Suicide and fall were increased from $6.6 \%$ to $27.0 \%, 8.3 \%$ to $11.8 \%$ respectively in 20 years. 
Conclusions Although the injury mortality has been decreased in the past 20 years, injury is still a public health problem in Shenzhen City. The road traffic injury in Shenzhen was still a main leading injury cause of death. Meanwhile, we should paid more attention on suicide and fall. Only do we change our preventive strategies, injury preventive measures should be more efficient.

\section{FRENCH HOME AND LEISURE INJURY PERMANENT SURVEY: WHAT CONTRIBUTION TO EPIDEMIOLOGICAL SURVEILLANCE?}

${ }^{1}$ Gaëlle Pédrono, ${ }^{2}$ Jean-Pierre Darlot, ${ }^{2}$ Marc Nectoux, ${ }^{1}$ Linda Lasbeur, ${ }^{1}$ Bertrand Thélot ${ }^{1}$ Santé Publique France, Saint-Maurice, France, ${ }^{2}$ Université Paris Descartes, Paris, France

\subsection{6/injuryprev-2016-042156.482}

Background The literature and the knowledge regarding home and leisure injury (HLI) are far wider than it used to be a couple of decades ago. Different types of epidemiological surveillance have been carried out: cross-sectional, population, cohort studies. Nevertheless, little is known about detailed circumstances of those injuries.

Methods The French HLI permanent survey started in France in 1986 in a dozen of hospital emergency services and is fully standardised since 2004. All patients admitted for HLI are included and detailed information is collected: where, when, how, who, what product is involved as well as the chronology of the injury and the care given.

Results In France, like in other countries, several systems provide epidemiological indicators to describe HLI: incidence in population, consumption of health care services, and people at risk. However, when a specific injury needs to be more documented, such as button battery ingestion, trampoline injuries, wasp stings, barbecue burns, traumatic sport injuries, elderly falls, etc., the only system able to provide solid answers in France is the HLI permanent survey, which records around 120000 annual injuries. The strength of the survey is the robustness guaranteed by the annual quality analysis and evaluations making the figures reliable and allowing time series analysis. Incidence rates can be estimated thanks to sophisticated models.

Conclusions For prevention purposes, there are increasing demands about HLI from the Ministry of Health, organisations, and associations. The HLI permanent survey is a key tool to improve knowledge about incidence, severity, preventability, and contributes to the diminution of those injuries through specific prevention initiatives and regulations.

\section{DOG BITES: SEVERITY AND SEQUELAE, A MULTICENTER SURVEY, FRANCE, SEPTEMBER 2010 - DECEMBER 2011}

${ }^{1}$ Gaëlle Pédrono, ${ }^{1}$ Cécile Ricard, ${ }^{1}$ Maryline Bouilly, ${ }^{2}$ Claude Beata, ${ }^{2}$ Guillaume Sarcey, ${ }^{1}$ Bertrand Thélot. ${ }^{1}$ Santé Publique France, Saint-Maurice, France; ${ }^{2}$ Association Des Vétérinaires Comportementalistes Zoopsy, France

\subsection{6/injuryprev-2016-042156.483}

Background Every year in France, dog bites result in several thousand admissions in emergency departments and numerous hospitalizations. The purpose of this survey was to identify severity factors and sequelae due to dog bites.

Methods An epidemiological study about the severity of $485 \mathrm{dog}$ bites was carried out in eight hospital emergency departments between 2009 and 2010; a study investigating sequelae 16 months after the bite was conducted among 298 of the 485 patients. Patients and dogs characteristics were collected by caregivers and veterinarians, 16-month sequelae were asked to patients by questionnaire. Multivariate regression analyses were conducted to explore severity and sequelae risk factors.

Results In children, bites were more frequent in the head and neck, but lesions were more severe in adults. The bites were more numerous and more serious when the victim knew the biting dog. No association was found between the severity of the bite and the type of biting dog. Almost half of respondents (47\%) reported sequelae; most of them were aesthetic (91\%). There were more sequelae when the bite was located at the head or inferior limbs; the sequelae were more frequent among women, when the weight of the biting dog was higher, and when the initial severity of the bite was higher. The link between the victim and the dog, the sex of the dog, the type of aggression, as well as the age of the victim, had no impact on the occurrence of sequelae. One patient out of seven was still having pain 16 months after the bite, women more often than men.

Conclusions Numerous patients still suffer 16 months after a dog bite, articles providing this type of results were not found in the literature. Dissemination of these results among professionals (physicians and veterinarians) and the general public should contribute to make dog owners aware of the risk of bite and the means to avoid them.

\section{STUDY ON CHILD HEAD INJURIES THROUGH DATA FROM THE NATIONAL INJURY SURVEILLANCE: SYSTEM OF CHINA, 2014}

Ji Cuirong, Er YuLiang, Ye Pengpeng, Wang Yuan, Deng Xiao, Gao Xin, Jin Ye, Wang Linhong, Duan Leilei. National Centre for Chronic and Noncommunicable Disease Control and Prevention, Chinese Centre for Disease Control and Prevention

\subsection{6/injuryprev-2016-042156.484}

Background Child head injury is an important public health issue. It is estimated that head injury is the first cause of child disability. The data from National Injury Surveillance System of China 2014 showed that about 200,000 cases went to outpatient and emergency due to head injury, $1 / 5$ of them were child aged 0 to 14 .

Methods Descriptive analysis was applied to depict general information, injuries events and clinical characteristics of child head injuries in 2014.

Results In 2014, 47690 cases of child head injuries were collected, males were twice higher than females, with $43.5 \%$ of them under 1-4 years of age. October, Thursday and 6 PM were the peak period of time. The three leading causes responsible for child head injuries were falls (69.6\%), blunt force injuries $(14.23 \%)$ and road traffic injury (11.0\%). Main locations causing head injuries were at home(45.0\%), public places (19.7\%), in road/street (15.8\%). Recreation activates (77.88\%), driving (7.32\%), sports activities (5.72\%) were the three major activities when injuries took place. The main injury intent was unintentional (95.4\%). The three leading nature of injury were bruise injuries (71.7\%), Sharp injuries, bites and open injuries (20.1\%), concussion/cerebral contusion (4.0\%). Most of the injury cases were mild $(90.3 \%)$, only $13.8 \%$ of them were moderate, and $0.9 \%$ of them were severe. The major disposition of injury cases were treated and discharged (82.0\%), others were observed/ admitted/transferred (9.4\%) and dead (0.04\%).

Conclusions In 2014, child head injuries were seen more in male than in female, mostly occurs at home. The leading causes for head injuries were falls, blunt injuries and road traffic. 